\title{
Practice and Knowledge of Contact Lens Use Among Medical Students of Universiti Teknologi MARA
}

\author{
Nor Azimah Abd Aziz ${ }^{1}$, Nurliyana Ain Abdul Ghani ${ }^{1}$, Khairil Anuar Md Isa ${ }^{2}$, Norasyikin Mustafa ${ }^{1}$ \\ 1 Department of Ophthalmology, Faculty of Medicine, \\ Universiti Teknologi MARA, Sg Buloh Campus, Selangor, Malaysia \\ ${ }^{2}$ Department of Health Sciences, Faculty of Health Sciences, \\ Universiti Teknologi MARA, Puncak Alam Campus, Selangor, Malaysia \\ azimah80@yahoo.com, nurliyanaain@yahoo.com, khairil_hpm@hotmail.com, norasyikinm@yahoo.co.uk \\ Tel: 03-61265000
}

\begin{abstract}
Contact lens is an optical device that is commonly used to correct refractive errors of the eye. Improper care may lead to ocular related complications This was a descriptive study to assess contact lens care practice and knowledge on contact lens use among medical students of Universiti Teknologi MARA (UiTM). Out of seven proper practices of contact lens care listed, only hand washing practice was adhered by most of the wearer. Both groups of contact lens wearer and non-wearers showed a moderate level of knowledge with regards to lens care. Educational programs on awareness and safety usage of contact lens should be emphasized particularly to the medical students
\end{abstract}

Keywords: Practice, Knowledge, Contact Lens, Medical Students

eISSN: 2398-4287 @ 2019. The Authors. Published for AMER ABRA cE-Bs by e-International Publishing House, Ltd., UK. This is an open access article under the CC BYNC-ND license (http://creativecommons.org/licenses/by-nc-nd/4.01. Peer-review under responsibility of AMER (Association of Malaysian Environment-Behaviou Researchers), ABRA (Association of Behavioural Researchers on Asians) and cE-Bs (Centre for Environment-Behaviour Studies), Faculty of Architecture, Planning \& Surveying, Universiti Teknologi MARA, Malaysia.

DOI: https://doi.org/10.21834/e-bpj.v4i11.1742

\subsection{Introduction}

Contact lens use has gained popularity among younger generations (Abahussin et al., 2014; Leeamornsiri and Titawattanakul, 2015; Yousef Bamahfouz et al., 2016). Its use is attractive to university students due to many reasons such as convenience, availability, and cosmetics (Zhu et al., 2018). Patient age influences lens wearing behaviours. Wearers of age 18-21 and those 22-25 years had been showed to have more non-compliance attitudes in contact lens care (Wagner et al., 2014). Although the contact lens has been widely used, many wearers are not aware that improper care and failure to follow the recommended lens care guidelines could increase the probability of serious eye complications.

The recent years' data do not show much result variation compared to studies done in previous decades in term of awareness and good contact lens practice. To create awareness regarding the proper use and care of contact lens, medical students need to educate themselves on the safe care practices as they will become the future health care providers, regardless they are contact lens wearers or non-wearers. Therefore, this study aims to determine the practice of contact lens usage and care and to assess the knowledge about contact lens care, including its related complications among medical students of Universiti Teknologi MARA (UiTM).

\subsection{Literature Review}

\subsection{Use of Contact Lens among University Students}

eISSN: 2398-4287 @ 2019. The Authors. Published for AMER ABRA cE-Bs by e-International Publishing House, Ltd., UK. This is an open access article under the CC BYNC-ND license (http://creativecommons.org/licenses/by-nc-nd/4.0/). Peer-review under responsibility of AMER (Association of Malaysian Environment-Behaviour Researchers), ABRA (Association of Behavioural Researchers on Asians) and cE-Bs (Centre for Environment-Behaviour Studies), Faculty of Architecture, Planning \& Surveying, Universiti Teknologi MARA, Malaysia. DOI: https://doi.org/10.21834/e-bpj.v4i11.1742 
Contact lens use among the younger generation particularly the university students is increasing with predominant female wearers. Prevalence of use among university students range from 20\%-50\% (Bamahfouz et al., 2016; Zhu et al., 2018) whilst prevalence of contact lens wear among the medical students varied from 14-27.4\% (Giri, Chavan, Phalke, \& Bangal, 2012; Tajunisah, Ophth, Reddy, \& Phuah, 2008) Recent studies demonstrated a higher prevalence of contact lens wear among the non-medical students and medical students (53.5\%, 32.2\% respectively)(Leeamornsiri \& Titawattanakul, 2015). Ibrahim et al reported that the prevalence of contact lens use among medical students in one of the universities in Pakistan was $40.5 \%$. Almost all of the above studies reported that the commonest reason for wearing contact lens is cosmetic. Despite being regarded as an educated population, these studies have shown that the contact lens wearers had limited knowledge about using and care of contact lens.

\subsection{Complications Associated with Contact Lens Use}

Even though contact lens is generally safe to use, it is not without risks. Complications of contact lens wear range from noninfective to infective. The former includes corneal neovascularization, dry eyes, giant papillary conjunctivitis, pinguecula, superior epithelial arcuate lesion, ptosis, and deep stromal opacity (Alipour, Khaheshi, Soleimanzadeh, Heidarzadeh, \& Heydarzadeh, 2017). Infective complications namely microbial keratitis imposes potentially serious complications with significant visual impairments. Contact lensrelated keratitis is due to active inflammation of the cornea caused by either bacteria, viruses, fungus or parasites. (Shah et al., 2011). Fungal keratitis is the major cause of microbial keratitis in certain developing countries (Acharya, Acharya, \& Karki, 2017) Meanwhile, bacterial keratitis is mainly caused by Pseudomonas (Chin, Young, Hui, \& Jhanji, 2015; Stapleton \& Carnt, 2012). Approximately one out of five hospitalized cases of contact lens-related microbial keratitis requires corneal transplant (Hoddenbach et al., 2014).

\subsection{Risk Factors of Developing Contact Lens-related Complications}

Risk factors associated with microbial keratitis in contact lens wearers are multifactorial These can be divided into modifiable and nonmodifiable risk factors (Zimmerman, Nixon and Rueff, 2016). The former includes overnight or extended-wear of soft contact lenses, poor storage case hygiene and infrequent case replacement, smoking, lack of handwashing and purchasing lenses on the internet. Meanwhile, non-modifiable risk factors are wearing lenses for less than six months, male gender, socioeconomic status, and possibly a genetic predisposition

Contact lens can provide a surface for microbial growth in situ. During contact lens wear, subclinical inflammatory reaction and accumulation of neutrophil debris occur under the posterior lens surface. Subsequently, certain invasive strains of Pseudomonas aeruginosa and other bacteria have the ability to utilize this neutrophil-derived cellular debris to facilitate colonization of the lens (Patel, Hinojosa, Zhu, \& Robertson, 2018). P. aeruginosa has also been shown to form biofilms on the posterior surface of the lens. Besides, over time during contact lens use, posterior lens tear fluid can lose antimicrobial activity, thus increasing the risk of microbial keratitis (Wu, Zhu, Tam, Evans, \& Fleiszig, 2015).

\subsection{Methodology}

A cross-sectional study was conducted at the Faculty of Medicine of UiTM from June 2016 to July 2016, in which 208 medical students from Year 1 to Year 5 were selected using a convenience sampling method. Year 1 and Year 2 constituted pre-clinical students. This study received ethical approval from the Research Ethics Committee UiTM (Ref. No: 600-IRMI (5/1/16) and was conducted in accordance with the Declaration of Helsinki for human research. They were divided into two groups of contact lens wearers (CL) and non-contact lens wearers (NCL). They were considered as contact lens wearer if they have ever worn contact lens for any period of time. A self-administration of modified, pre-tested and validated questionnaire was used in this study to inquire about their practice and knowledge of the contact lens use (Tajunisah et al., 2008). The questionnaire was divided into three sections; A: Demographic data and details on background use of contact lens which include the purpose of use, duration of lens been used (in year), regularity of usage and duration of use per day. Section B comprises of seven questions on the practice of contact lens use, and C: Knowledge of contact lens use and care. The results were analysed using SPSS software (version 20.0, International Business Machines Corp.). Descriptive statistics were used to describe the data. Frequency and percentage were used for qualitative variables. Categorical data were analysed using chi-square test. The significant level was set at $\alpha<0.05$.

\subsection{Findings}

The demographic profile of both study groups is listed in Table 1. Out of 208 medical students, $79(38 \%)$ and $129(62 \%)$ were CL and NCL wearers, respectively. Almost $90 \%$ were female $(71,89.9 \%)$, with female to male ratio is almost $9: 1$. More than half of the wearers $(43,54.5 \%)$ were in the preclinical years. The duration of contact lens wear varied from less than a year to more than five years. The main reasons for wearing contact lens were refractive errors (58.2\%) and cosmetic purposes $(29.1 \%)$. Most wearers used contact lens occasionally $(41,51.9 \%)$ and only $21(26.6 \%)$ wore contact lens daily. About half of the wearers $(51.9 \%)$ wore contact lens less than eight hours a day.

Table 1: Demographic Profile

\begin{tabular}{lcc}
\hline Variable & CL Wearers & NCL Wearers \\
& $n(\%)$ & $n(\%)$ \\
\hline Sex & & \\
\hline
\end{tabular}




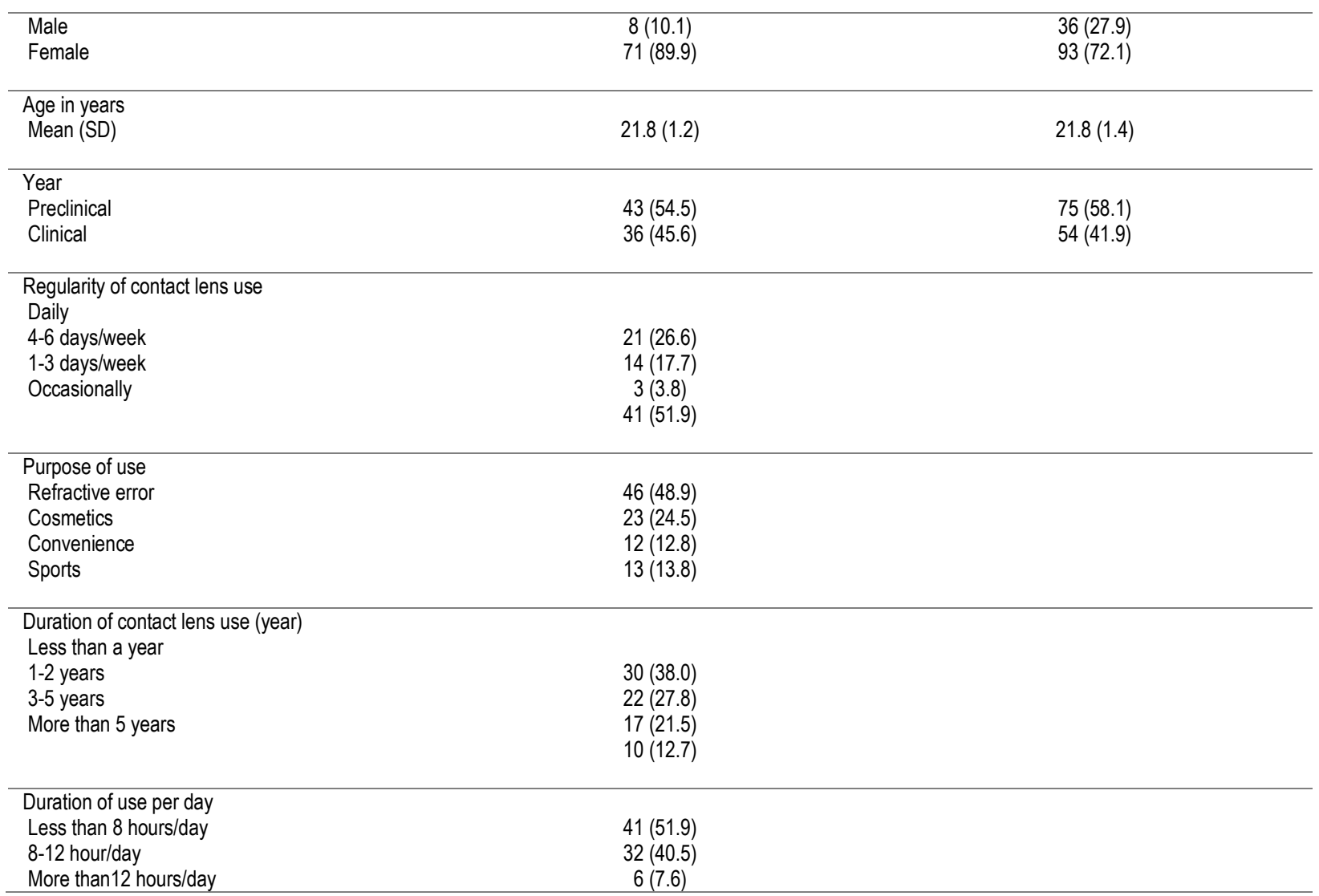

The knowledge of contact lens usage and care are shown in Table 2. The majority of the students from both groups ( $80 \%$ and $86 \%$ of $\mathrm{CL}$ and NCL wearers, respectively) knew that the contact lens should be prescribed by certified eye care providers $(\mathrm{p}=0.49)$. More than two-thirds of the wearers and non-wearers $(79.7 \%$ and $86 \%$ respectively) agreed that initial eye examination is required before using contact lens $(p=0.08)$. Less than half of the wearers agreed that a yearly eye examination is necessary when using contact lens compared to $72 \%$ in non-wearers ( $p<0.001)$. The majority of wearers and non-wearers $(96.2 \%$ and $94.6 \%$, respectively) knew that hand wash is required before and after lens insertion and removal $(\mathrm{p}=0.27)$. Around $83 \%$ and $79 \%$ of wearers and non-wearers, respectively, knew that the lens should be rubbed and rinsed with lens solution daily $(p=0.38)$. More than half of the students from each group did not know that there should be regular enzymatic cleaning of their lenses every month $(p=0.91)$.

Only one-third of the wearers knew that a visual inspection of the lens should be performed daily compared to $43.8 \%$ in non-wearers $(p=0.14)$. The majority of the students knew that contact lens wearers should not sleep with contact lens on. More than $90 \%$ of wearers knew that water should not be used as a storage solution as compared to non-wearers $(67 \%),(p<0.001)$. Only two-thirds of the students from each group aware that contact lens casing should be cleaned or disinfected daily ( $C L=57.1 \%, N C L=65.1 \%, p<0.001$ ).

Regarding the knowledge of contact lens wear complications, $89.9 \%$ and $84.5 \%$ of wearers and non-wearers respectively were aware of complications that are associated with improper handling of contact lens $(p=0.46)$. The majority of them were aware of other common contact lens-related complications such as allergic conjunctivitis and dry eyes. However, only half of the students from both groups knew about the occurrence of corneal ulcer with contact lens usage $(p=0.79)$.

Table 2: Knowledge of Contact Lens Usage and Care

\begin{tabular}{lccc}
\hline Variable & $\begin{array}{c}\mathrm{CL} \text { wearer } \\
\mathrm{n}(\%)\end{array}$ & $\begin{array}{c}\mathrm{NCL} \text { wearer } \\
\mathrm{n}(\%)\end{array}$ & p-value \\
\hline $\begin{array}{l}\text { Lens should only be prescribed by a certified eye } \\
\text { care provider }\end{array}$ & & & \\
Yes & $63(79.7)$ & $111(86.0)$ & 0.49 \\
No & $6(7.6)$ & $7(5.42)$ & $11(8.52)$ \\
don't know & $10(12.7)$ & &
\end{tabular}




\begin{tabular}{lcc}
\hline The eye examination is necessary before using & & \\
contact lens for the first time & $59(74.7)$ & $112(86.8)$ \\
Yes & $6(4.7)$ & $4(3.1)$ \\
No & $14(17.7)$ & $13(10.1)$ \\
Don't know &
\end{tabular}

\section{Yearly examination is necessary when using contact lens}

Yes

$36(45.6)$

No $4(5.1)$

$93(72.1)$

$6(4.7)$

$30(23.3)$

$<0.001$

Don't know

$39(49.4)$

$4(3.1)$

The hand should be washed before and after lens

insertion and removal

Yes

No $76(96.2)$

Don't know $1(1.3)$

$2(2.5)$

$122(94.6)$

$0(0.0)$

$7(5.4)$

\begin{tabular}{lcc}
\hline The lens should be rubbed and rinsed with lens & \\
solution daily & $66(83.5)$ & $101(78.3)$ \\
Yes & $1(1.3)$ & $6(4.7)$ \\
No & $12(15.2)$ & $22(17.1)$ \\
Don't know & & \\
& & $50(38.8)$ \\
\hline There should be regular enzymatic cleaning of lens & & $2(1.6)$ \\
(monthly) & $28(35.4)$ & $76(58.9)$ \\
Yes & $1(1.3)$ & $48(60.8)$ \\
No & &
\end{tabular}

Visual inspection of lens should be done daily
Yes
No
Don't know

Not to sleep overnight while wearing contact lens

Yes

No

Don't know

75 (94.9)

1 (1.3)

$18(14.0)$
$54(41.9)$

0.14

2 (2.5)

$2(1.6)$

$6(4.7)$

\begin{tabular}{lcc}
\hline Water should not be used as a storage solution & & \\
Yes & $72(91.1)$ & $87(67.4)$ \\
No & $3(3.8)$ & $4(3.1)$ \\
Don't know & $3(3.8)$ & $37(28.7)$
\end{tabular}

The lens container should be cleaned/ disinfected
daily
Yes
No




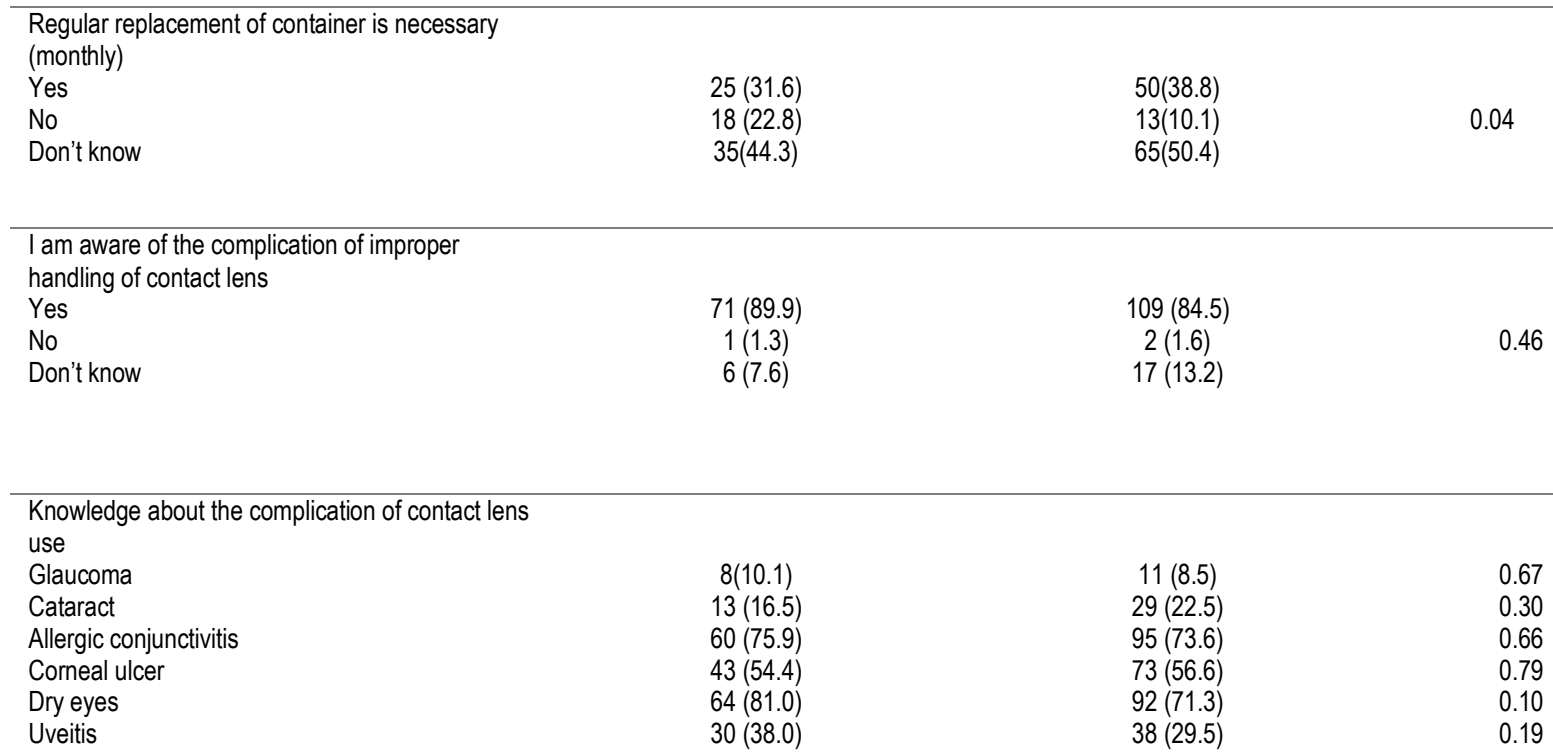

\section{Chi Square test.}

Findings on the practice of contact lens use among the wearers are displayed in Figure 1. Most of the $\mathrm{CL}$ wearers $(70,88.6 \%)$ wash their hands before handling the lenses and never let tap water into contact with contact lens $(59,74.7 \%)$. Less than half $(48.1 \%)$ of wearers claimed to never sleep with contact lens on, whereas $3.8 \%$ admitted they always did. The majority of the wearers never change contact lens solution daily and admitted never adhere strictly to contact lens cleaning instruction (75.9\% and $72.2 \%$ respectively). More than half $(43,54.4 \%)$ of the wearers never change the contact lens every month. Less than half $(35,44.3 \%)$ stated they would never wear contact lens when they experience blur vision eye pain when using lens. Two $(2.5 \%)$ of them would continue wearing their lens despite the symptoms. There was no significant difference between male and female wearer with regards to practice and care of contact lens.

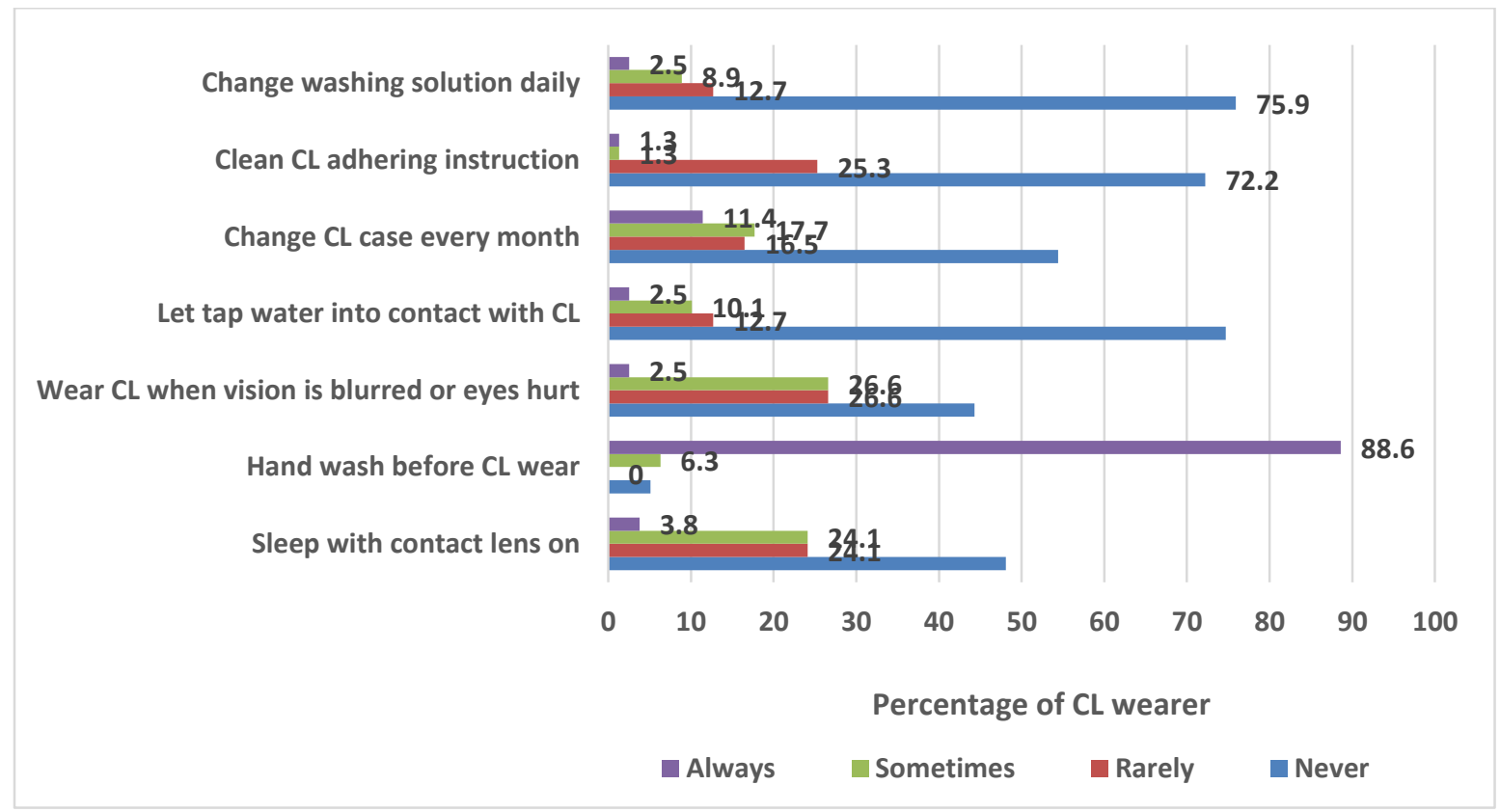

Figure 1: Practice of Contact Lens Use. Only hand washing was mostly adhered by the wearer 


\subsection{Discussion}

To the best of our knowledge, this is the first study of knowledge and practice on contact lens wear and care in both CL wearers and non-wearers among medical students. The purpose of this study was to assess how adequate they are as the future health care providers with the knowledge in contact lens care, regardless they are direct lens wearer or not.

Contact lens is considered a medical device. In Malaysia, even though contact lens usage requires a prescription and proper fitting by certified practitioner namely registered optometrist or optician with contact lens license, many are sold without following set rules ("The Risk Of Wearing Contact Lens From The Uncertified Practitioner - PORTAL MyHEALTH," 2017). About 80\% of contact lens users in our study knew the requirement of such prescription. About one-quarter of our CL users wear CL for cosmetic purposes. In China, $57.9 \%$ wore contact lens for aesthetic purposes (Zhu et al., 2018). Coloured cosmetic contact lenses, in particular, are readily available from online stores or over-the-counter. Usage of such decorative lens is usually unmonitored without prescription and can be associated with serious sight-threatening complications (Singh, Satani, Patel, \& Vhankade, 2012). It has been demonstrated that Acanthamoeba showed a greater affinity for the cosmetic contact lens (Lee, Lee, Lee, \& Yu, 2018)

The most devastating complication associated with contact lens use is microbial keratitis or corneal ulcer. Risk factors for contact lens-related corneal ulcer include not washing hands with soap before handling lenses, not performing rubbing technique, not cleaning lens cases with multipurpose solution daily and sleeping overnight wearing contact lens (Ismail et al., 2016). Out of seven proper practices of contact lens care listed in this study, only hand washing practice was adhered by most of the wearer. About $3.8 \%$ of wearer did not perform or know that they should wash their hands before handling contact lenses. The number of not knowing that rubbing and rinsing the lens with the multipurpose solution is important was higher (16.5\%). This knowledge among CL and NCL users was comparatively about similar ( $83.5 \%$ and $78.3 \%$, respectively, $p=0.38)$.

More than $93 \%$ of all students aware that users should not be sleeping overnight while wearing contact lens. However, more than half of the users had slept overnight without removing the contact lens. Our study demonstrated some discrepancies between knowledge on contact lens use and their actual practice which is similar to another study (Bui, Cavanagh, \& Robertson, 2010). Perhaps, hectic lifestyle among medical students and the ability to tolerate mild contact lens-related problems might influence this behaviour. Further study is required to determine the actual reasons. Extended wear CL beyond recommended use could increase the risk of microbial keratitis as the amount of oxygen getting to the cornea is reduced and may lead to corneal damage (Dumbleton, Woods, Jones, \& Fonn, 2013).

The majority of lens wearers knew that water should not be used as a storage solution, in contrast, a significant number of non-users were not aware of this $(92.3 \%$ vs. $68 \%, p<0.001)$. A quarter number of $C L$ wearers had ever let tap water into contact with their contact lenses. This is a dangerous practice as associations of Acanthamoeba keratitis and improper contact lens hygiene has been wellestablished particularly contact with water (Ma \& Rhee, 2018). Regarding the yearly eye examination while wearing contact lens, there was a significant difference between the two groups; $45 \%$ of the CL wearer did not find it necessary but $72 \%$ of the NCL wearer agreed. Being asymptomatic is probably the cause of this finding among the lens users. Furthermore, lack of information acquired during the initial lens prescription could be a factor. However, this was not explored in our study.

The majority of lens wearers and non-wearers claimed that they were aware of complications associated with improper lens use. Ironically, less than half of $\mathrm{CL}$ wearers continue wearing contact lens despite having eye symptoms such as blurring of vision and pain. This truly reflects their knowledge of the complication of contact lens use. Majority of them were aware of dry eyes symptoms but only half of the group knew the possibility of getting corneal ulcer. A study showed that almost all lens wearing patients exhibit behavioural non-compliance despite considering themselves to be complying with standard guidelines for lens wear and care practices (Robertson \& Cavanagh, 2011). These findings showed that perceived compliance, awareness or knowledge in lens care is not an indicator of appropriate patient behaviour (Bui et al., 2010).

Proper contact lens care and handling are essential to ensure comfort and to avoid contact lens-related complications. Recommendation of proper care of contact lens include washing and drying the hands prior to lens handling, rubbing and rinsing the lenses with contact lens disinfecting solution, rubbing and rinsing the lens case with washing solution after use, to dry the case with a clean tissue and to store it upside down with the caps off after each use, to soak the lenses overnight in fresh solution, to remove lenses before sleeping and to avoid lens contact with tap water (Razmaria, 2015). These proper practices can be improved by giving a detailed explanation and behaviour modification upon the prescription of CL (McMonnies, 2011). Most users may be non-compliant because they do not have an adequate practical and/or scientific basis for the usage. This was particularly true in pre-clinical students who have inadequate clinical exposure and have yet to be exposed to ocular diseases. Apart from that, it may be due to the wearer's ignorance towards the importance of proper contact lens usage and care.

Our study limitation is the types of contact lens used by the participants were not explored in this current study. This might contribute a bias response from the daily-disposable $\mathrm{CL}$ user when answering questions on the practice of contact lens use. For instance, the question of changing the lens case every month. A daily-disposable CL user usually will discard the container daily after each use. Nevertheless, our study overall provides useful information in determining the weight of this current issue in our study population. Further research in the psychological or behavioural study, for instance, using a health belief model is required to explore the reasons for poor compliance in contact lens care particularly among medical students.

\subsection{Conclusion \& Recommendation}

The results of this study have shown an unsatisfactory level of knowledge among medical students indicated by moderate knowledge level and poor practices related to contact lens wear, care, and complications. There is a need for more education, awareness and 
behavioural program to contact lens wearers especially medical students regarding contact lens usage, care and its related complications, which should be provided by all primary eye care practitioners. Medical students will be the future possible opinion formers and communicators of knowledge related to contact lens and prevention of complications.

\section{Acknowledgement}

We would like to express our sincere gratitude to undergraduate medical students (Group 10-2016) for their contributions in this study.

\section{References}

Abahussin, M., AlAnazi, M., Ogbuehi, K. C., \& Osuagwu, U. L. (2014). Prevalence, use and sale of contact lenses in Saudi Arabia: Survey on university women and nonophthalmic stores. Contact Lens and Anterior Eye, 37(3), 185-190.

Acharya, Y., Acharya, B., \& Karki, P. (2017a). Fungal keratitis: study of increasing trend and common determinants. Nepal Journal of Epidemiology, 7(2), 685-693.

Alipour, F., Khaheshi, S., Soleimanzadeh, M., Heidarzadeh, S., \& Heydarzadeh, S. (2017). Contact Lens-related Complications: A Review. Journal of Ophthalmic \& Vision Research, 12(2), 193-204.

Bui, T. H., Cavanagh, H. D., \& Robertson, D. M. (2010). Patient compliance during contact lens wear: perceptions, awareness, and behavior. Eye \& Contact Lens, 36(6), $334-339$.

Chin, J., Young, A. L., Hui, M., \& Jhanji, V. (2015). Acanthamoeba keratitis: 10-Year study at a tertiary eye care center in Hong Kong. Contact Lens and Anterior Eye, 38(2), 99-103.

Dumbleton, K., Woods, C. A., Jones, L. W., \& Fonn, D. (2013). The Impact of Contemporary Contact Lenses on Contact Lens Discontinuation. Eye \& Contact Lens: Science \& Clinical Practice, 39(1), 92-98.

Giri, P. a, Chavan, W. M., Phalke, D. B., \& Bangal, S. V. (2012). Knowledge and Practice of Contact Lens Wear and Care Among Contact Lens Users Medical Students of Rural Medical College , Loni , Maharashtra, India. International Journal of Biological \& Medical Research, 3(1), 1385-1387.

Hoddenbach, J. G., Boekhoorn, S. S., Wubbels, R., Vreugdenhil, W., Van Rooij, J., \& Geerards, A. J. M. (2014). Clinical presentation and morbidity of contact lensassociated microbial keratitis: a retrospective study. Graefe's Archive for Clinical and Experimental Ophthalmology, 252(2), 299-306.

Ismail, L. A., Rampal, L., Rahman, H. A., Omar, N., Midi, H., \& Ariffn, A. E. (2016). Risk factors associated with contact lens related microbial keratitis. Malaysian Journal of Medicine and Health Sciences, 12(1), 1-8.

Lee, S. M., Lee, J. E., Lee, D. I., \& Yu, H. S. (2018). Adhesion of Acanthamoeba on Cosmetic Contact Lenses. Journal of Korean Medical Science, 33(4)

Leeamornsiri, S., \& Titawattanakul, Y. (2015). Comparative knowledge and behavior of contact lens care between medical and non-medical students. Journal of the Medical Association of Thailand, 98, S16-S23.

Ma, L., \& Rhee, M. K. (2018). Contact Lenses and Infectious Keratitis. Current Ophthalmology Reports, 6(2), 115-125.

McMonnies, C. W. (2011). Improving contact lens compliance by explaining the benefits of compliant procedures. Contact Lens and Anterior Eye, 34(5), 249-252.

Patel, N. B., Hinojosa, J. A., Zhu, M., \& Robertson, D. M. (2018). Acceleration of the formation of biofilms on contact lens surfaces in the presence of neutrophil-derived cellular debris is conserved across multiple genera. Molecular Vision, 24, 94-104.

Razmaria, A. A. (2015). Proper care of contact lenses. JAMA - Journal of the American Medical Association, 314(14), 1534

Robertson, D. M., \& Cavanagh, H. D. (2011). Non-compliance with contact lens wear and care practices: a comparative analysis. Optometry and Vision Science : Official Publication of the American Academy of Optometry, 88(12), 1402-1408.

Shah, A., Sachdev, A., Coggon, D., \& Hossain, P. (2011). Geographic variations in microbial keratitis: an analysis of the peer-reviewed literature. The British Journal of Ophthalmology, 95(6), 762-767.

Singh, S., Satani, D., Patel, A., \& Vhankade, R. (2012). Colored Cosmetic Contact Lenses. Cornea, 31(7), 777-779.

Stapleton, F., \& Carnt, N. (2012). Contact lens-related microbial keratitis: how have epidemiology and genetics helped us with pathogenesis and prophylaxis. Eye (London, England), 26(2), 185-193.

Tajunisah, I., Ophth, M., Reddy, S. C., \& Phuah, S. J. (2008). Knowledge and practice of contact lens wear and care among medical students of University of Malaya. The Medical Journal of Malaysia, 63(3), 207-210.

The Risk Of Wearing Contact Lens From The Uncertified Practitioner - PORTAL MyHEALTH. (2017). Retrieved May 29, 2019, from http://www.myhealth.gov.my/en/riskwearing-contact-lens-uncertified-practitionerl

Wagner, H., Richdale, K., Mitchell, G. L., Lam, D. Y., Jansen, M. E., Kinoshita, B. T., ... Chalmers, R. L. (2014). Age, behavior, environment, and health factors in the soft contact lens risk survey. Optometry and Vision Science, 91(3), 252-261.

Wu, Y. T., Zhu, L. S., Tam, K. P. C., Evans, D. J., \& Fleiszig, S. M. J. (2015). Pseudomonas aeruginosa Survival at Posterior Contact Lens Surfaces after Daily Wear. 
Optometry and Vision Science : Official Publication of the American Academy of Optometry, 92(6), 659-664.

Yousef Bamahfouz, A., Nafady-Hego, H., Jouhargy, S., Qadir, M. A., Nabeel, W., Qutub, J., ... Al-Ghamdi, A. A. (2016). Awareness of Contact Lens Care among College Students in Saudi Arabia. International Journal of Scientific Study, (1).

Zhu, Q., Yang, B., Deng, N., Li, Y., Wang, T., Qi, H., \& Liu, L. (2018). The use of contact lenses among university students in Chengdu: Knowledge and practice of contact lens wearers. Contact Lens and Anterior Eye, 41(2), 229-233. 